\title{
What would most help improve the quality of life of older family carers of people with dementia? A qualitative study of carers' views
}

\section{Abstract}

Older family carers of people with dementia provide a substantial amount of care for people with dementia in the UK. Caregiving can be stressful and burdensome for these individuals, who are also experiencing psychological and physical changes resulting from their own ageing process. However, little is known about what impacts their quality of life, how this can be improved and what we should prioritise. This brief report asks one simple question to older family carers of people living with dementia - "What would most help improve your quality of life as a carer?" Qualitative data was collected from 150 carers who completed an anonymous paper survey during the development and validation of a quality of life tool for use with this population (DQoL-OC). Participants were individuals aged 60 and over and were providing care for a family member with dementia at home in the UK. Carers were recruited from a variety of voluntary organizations, community-based carers' groups, health services and via online forums. A thematic approach was used to analyse the carers' comments and three main overarching themes were identified. The quality of life of older family carers can be enhanced by having more time away from caregiving, accessing health and social services that are dementia friendly and by having economic support. Future care, policies and research should aim to address these key areas in order to promote better quality of life for older carers of people with dementia. Further implications for practice, policy and research are discussed.

\section{Keywords}

dementia, older family carers, caregivers, quality of life, qualitative study, thematic analysis 


\section{Introduction}

The UK annual cost of dementia is estimated to be of $£ 26.3$ billion, which is equivalent to $25 \%$ of the UK total annual health budget (Prince et al., 2014; NHS, 2015). Of this, only £8.8 billion has been contributed by the UK Government, with the remaining $£ 17.4$ billion coming from people with dementia and their family members (Kane and Terry, 2015). Family carers are therefore a key source of support for people with dementia living in the community within the UK and we should find better ways to support them (HM Government, 2009).

A great proportion of family carers are older people who are living with physiological and physical changes as a result of their own ageing process (Carers Trust, 2011; White, 2013). Carers aged $70+$ are responsible for proving care for the longest periods of time, often in excess of 60 hours per week, seven days a week (Carers Trust, 2011). These individuals usually co-reside with their loved one and care is provided full-time and with no respite breaks (Steptoe et al., 2015). These carers often report poorer mental and physical health outcomes when compared with young adult carers or age-matched controls (Butterworth et al., 2010; Huang, 2012) and have a higher risk of general psychiatric morbidity (Al-Zahrani et al., 2015). Caregiving can lead older carers to a decline in self-care (Gallant and Connel, 1997), poor family relationship quality (Quinn et al., 2009), higher levels of depression (Covinsky et al., 2003) and higher risk of deterioration in cognitive function (Vitaliano et al., 2011).

Despite these consequences, there is currently a dearth of research on how the Quality of Life (QoL) of older family carers of people with dementia can be improved (Oliveira et al., 2015; Lautenschlager, 2013). QoL is a multidimensional construct that provides useful information for researchers and social and health care professionals seeking a better understanding of the impact of health conditions on a wide range of life aspects such as physical health, psychological state, level of independence, social relationships, personal beliefs, and relationship with the environment in which they live (Fayers and Machin, 2016). Within the extant literature, only four studies investigating the QoL of older family carers (aged 60+) of people with dementia have been identified (Oliveira et al., 2015). In a review of these papers, Oliveira et al (2015) found that QoL is lower in older people, with high levels of burden and more 
time committed to care, in those experiencing depression, in females and in those with more dependent care recipients. With such a paucity of research in this area, there is a need for further exploration that may allow us to understand what older carers' key priorities are in terms of life quality. This study therefore aimed to explore what older family carers of people with dementia believe would most improve their QoL.

\section{Method}

This qualitative single-item design formed part of an anonymous paper questionnaire used to develop and validate a QoL tool for older family carers of PwD (reference to be added after the manuscript is accepted). To take part in the research, individuals needed to be aged 60 and plus and to be providing unpaid care for a family member with dementia at home in the UK at the time of the study.

Carers were recruited from a variety of voluntary organizations, community-based support groups, general practices and memory clinics. Information about the study was also publicized in public areas and online. Potential participants either contacted the researcher directly from adverts or were approached by the researcher or support group leaders. Carers were offered information about the study and were asked to provide consent in order to take part.

The data analysed in this paper were collected via an open-ended question - "What would most help improve your quality of life as a carer?" The use of 'single questions' to evaluate people's QoL has been shown to produce valid and reliable QoL scores in quantitative studies (Boer et al., 2004) and it was anticipated that a qualitative approach using a similar method of enquiry would elicit useful information about what is important for the QoL of carers aged 60+.

Ethical approval was obtained from the National Research Ethics Committee service and appropriate NHS R\&D committees in the UK (15/IEC08/0041). Participant information was provided and written consent obtained from carers before entering the study. Data collection was carried out in line with the Declaration of Helsinki from 1964 (World Medical Association, 2013). 


\section{Characteristics of the sample}

Carers taking part in the study $(n=150)$ had a mean age of 72.15 years old ( $S D=$ 8.31 ), with $32.2 \%$ aged $80+$, and were mostly females (65\%), married ( $89.5 \%)$, white $(96 \%)$, and had no qualification (28\%). Individuals were predominantly spousal carers $(80 \%)$, living with their loved ones with dementia (83\%) and had been providing care for between 1 and 6 years (78\%). About $55 \%$ of carers were providing care for more than 12 hours a day and $89 \%$ provided care 6 to 7 days a week.

\section{Data analysis}

The data collected via the paper questionnaires were typed to an excel file and then analysed thematically (Braun and Clarke, 2006) by two researchers (DO and LS) independently, following an inductive approach. First, the dataset was thoroughly explored aiming to identify repeated patterns of meaning (codes). The analysis was carried out on an explicit/ semantic level (Braun and Clarke, 2006), nonetheless a conscious exercise was made to consider possible alternative readings and exploring variation and even contradictions. The researchers were mindful of the reflexive nature of this qualitative research (Macbeth, 2001) and tried to avoid any conclusion based on personal assumptions (Frith and Gleeson, 2004). After an initial coding, a poll of themes was identified and collated as part of a broad thematic map. The central ideas and concepts of each theme then guided the creation of overarching themes. A final set of themes and overarching themes was agreed upon after several discussions and consensus among the research team, following an iterative process.

\section{Results}

Data clustered around three overarching themes: 1) More opportunities to enjoy life outside caregiving; 2) Carer/dementia friendly health and social care systems; and 3) Financial support (see Table 1). The first overarching theme represents the reasons why the older carers need more time away from caregiving and what support mechanisms there needs to be in place for that to occur. The second one represents the importance of having understanding, knowledgeable and empathetic professionals available to them, as well as the importance of having social 
relationships with people who are dementia aware. The third and final overarching theme represents the importance of receiving financial support and having flexible

Table 1. Overarching themes and sub-themes

\begin{tabular}{llc}
\hline Overarching theme & Theme & $\begin{array}{c}\text { Frequency of } \\
\text { occurrence }\end{array}$ \\
\hline 1. More & 1. Time for themselves & 39 \\
opportunities to & 2. Appropriate care alternatives in place & 30 \\
enjoy life outside & 3. Sharing responsibilities & 7 \\
caregiving & 4. Support from family and friends & 7 \\
2. Carer/dementia & 5. Quality time with the PwD & 6 \\
friendly health and & 7. Accessible Services & 23 \\
social care systems & 8. Proximity of care & 26 \\
& 9. Care for themselves and information & 10 \\
& 10. Public awareness and understanding & 8 \\
3. Financial support & 11. Financial support & 15 \\
& 12. Flexibility at work & 3 \\
Total & 2. & 188 \\
\hline
\end{tabular}

work policies in place so that the older carers can maintain a good quality of life.

\section{More opportunities to enjoy life outside caregiving}

Participants expressed the need to have more time away from caregiving (theme 1) in order to be able to rest, to pursuit their personal goals, to maintain their social life and identity. This was the most frequent sub-theme identified in the entire dataset.

"Give me a bit more breathing space"

"More time do my own things"

"Occasionally being able to have a break and not having to worry about the person I care about" 
Oliveira, Sousa \& Aubeeluck 2018 (accepted for publication - 'Dementia' SAGE on 4 Jul 2018)

This lack of time to themselves led to a difficulty in finding a balance between their own lives and caring, which led them to experience a sense of loss and lack of selfrealization and purpose in life.

"I feel I have lost so much of my future. It's all swallowed up by caring and I will never realise my dreams"

"If I am doing anything for myself I am never left to finish it without having to deal with something my husband needs".

Despite recognising their need for respite, carers admit that, depending on the quality and timing of this support, they may prefer not to have it, as sometimes it can be more laborious than beneficial to them (theme 2).

"My main problem is respite periods when strange carers come in and live in while I'm away. This period varies greatly from good care to abysmal and I sometimes wonder if it's worth having respite"

"What would help was to have some time apart from the person that I care for, knowing that he was happy"

"My problem is that I am reluctant to share the burden with anyone else. I realise that perhaps at some time I would have to"

"Respite care and day care need to be more readily available. Respite care needs to be bookable so that carers can book and plan a holiday rather than just taking it at short notice as it becomes available"

Older carers also felt that being able to share the decision-making process and the overall caring responsibilities (theme 3 ) would help them to enjoy life outside their caregiving role: 
Oliveira, Sousa \& Aubeeluck 2018 (accepted for publication - 'Dementia' SAGE on 4 Jul 2018)

"I feel I am alone taking all decisions with no one to lean on, except my daughter"

"To be free from the tensions of the necessary routine of everyday life. The responsibility, for [the life/happiness] of someone once loved who cannot understand or appreciate their illness or needs"

Many older family carers also believed that more support from family and friends could allow them to have a break from care have more quality of life (theme 4).

"More help from family members"

"Support and help from close family would make a big difference"

More than respite, many spousal older carers wanted to spend quality time with their loved ones as they used to do in the past, not just in a caring capacity (theme 5). It seemed difficult for them to accept that the relationship dynamic had changed and the presence of dementia symptoms (e.g. anxiety) seemed to be a limiting factor.

"Being able to go on holiday with my wife as before, but the anxiety part of the illness would not allow it"

"If my wife were to enter a more passive phase and go along with my suggestions (e.g. go on holiday) it would vastly improve the quality of both our lives".

\section{Carer/dementia friendly health and social care systems}

Several older carers disclosed how their experience at the dementia diagnosis was upsetting and that services did not provide support that they needed at the time (theme 6). This was particularly scary and caused a sense of helplessness to some participants, who believe that more training is needed for staff to enhance the quality of the services for them. 
Oliveira, Sousa \& Aubeeluck 2018 (accepted for publication - 'Dementia' SAGE on 4 Jul 2018)

"When [name] was diagnosed, we came out of the clinic thinking what do we do now, all they said to us they would refer us to our GP. Nothing to say what sort of treatment or help we would get. Very lonely place to be, we felt very much alone [...] All we seem to get is take the tablets and the review date every six months everyone gets from their GP. We felt very much alone. Frightened."

"If social and health services at least had some basic training in dementia".

The older family carers in this study expressed the need of health and social care systems that were more flexible and easier to access, so that they would not need to worry about finding the available services to have their needs met (theme 7).

"Appointments with my GP whom I am registered with seems impossible, always full"

"Easier access to departments relating to my needs"

"More communication between the care service and myself - I shouldn't have to chase them"

"Appointments for my mother always seem to be at the convenience of the professionals - not me"

Carers reported that lack of funding and high pressure on services left them without the support needed to meet their needs, particularly regarding long-term specialized care (theme 8). This compromised how close they could be from professionals and the continuity of the care provided long-term.

"I miss the proximity, monthly visit from CPN [Community Psychiatric Nurse] which we enjoyed until last year. The service was withdrawn from us because of pressure from the demand for new referrals... The visits gave me some reassurance that I was doing OK as a carer, and talking to a GP (however sympathetic) is not quite the same." 
Oliveira, Sousa \& Aubeeluck 2018 (accepted for publication - 'Dementia' SAGE on 4 Jul 2018)

In addition to the care provided to their relatives with dementia, the older carers in this study did not feel that their own needs were a priority for the health system and were largely unmet (theme 9). Some had not received the necessary training and knowledge to provide care. More support for their own needs and more information could greatly improve the quality of their lives.

"As a carer you become invisible as a person"

"I feel there should be more regular health checks, assessments etc. for the family member [carer]"

"No one has provided me with any information about dementia or caring for someone with dementia, I have had to learn by trial and error"

The older family carers also believe that there needs to be more public awareness and understanding toward dementia and family caring (theme 10). They suggested there should be more recognition and appreciation for all the work they do to society and the impact this has on both the person living with dementia and themselves as carers.

"Getting other people to understand that my husband has dementia and that it impacts on daily life"

"For people to appreciate what it involves"

"People to really understand what it takes and how much it takes out of a person to care $24 / 7$ "

\section{Financial support}

The third and final theme identified was about the financial constraints related to the costs arising with the caring needs. Carers did not just point out the need for provision of allowances (theme 11), but also the need for flexibility at work, allowing 
them to continue to work and be self-funded while caring for someone with dementia (theme 12).

"[a paid] carer at present [costs] over $£ 50$ a day fee, regular use is prohibitive to the average family"

"I am trying to juggle working full time and caring for my father, who lives 90 miles away. I would like to be able to work more flexibly so that I can have my father living with me"

The older carers in this study also worried that there would not be enough resources for them when they needed support with their own needs in the future.

"I pay for all my spouses' care, which will impact on my own future"

\section{Discussion}

Older family carers of people with dementia represent an increasing, but overlooked population of family carers in the UK, and little is known about what can be done to improve their QoL. To our knowledge, this is the first study that directly asks older family carers of people with dementia what would most improve their QoL providing them the opportunity to reflect on what is most important to them.

In considering the importance to carers of enjoying life outside of caregiving, carers report that having a full-time caring role leaves little space for them to enjoy activities with other people. Carers UK and Age UK (2015) suggest that older family carers of people with dementia are responsible for providing the highest number of hours and days a week of care leaving little time or space for their own lives and highly susceptible to social isolation. In addition, carers were often uncertain of the quality or appropriateness of care provision that could be put into place to provide respite and ameliorate the issue of having little time for themselves. They were seeking opportunities to share the burden of decision making with other family members, explore opportunities for additional support from within the family and spend time doing activities with the PwD that were not just caregiving related. 
The inability to draw these different threads of the same overarching theme of seeking more opportunities to enjoy life outside of caregiving leads to loneliness and isolation for many of these carers. Previous studies have demonstrated that isolation is associated with lower levels of well-being in this population (Raivio et al., 2015), that older spousal carers experience more loneliness (Lavela and Ather, 2010) and lonely older carers exhibit more depressive symptoms over time than those who do not feel lonely (Jaremka et al., 2014). A large study carried out in Sweden further demonstrated that high levels of loneliness are associated with lower QoL in older carers (Ekwall et al., 2005). Dilworth-Anderson (2015) argue that this may be caused by high levels of social activity conflict resulting from a demanding caregiving role, especially in dementia.

A number of other studies have also demonstrated how support from family and friends can help to decrease depressive symptoms (Moon and Dilworth-Anderson, 2015), burden (Coen et al., 2002), and loneliness (Ekwall et al., 2005). Moreover, having access to extensive social ties (Berkman et al., 2004), a productive role (Rozario et al., 2004), strengthened social networks (Huang, 2012), and more general resources (Ahn et al., 2012; Neri et al., 2012) are all associated with more positive health and better psychological outcomes. We would therefore argue that having time away from caregiving is likely to improve their psychological well-being, contributing to an overall better QoL.

In considering carer/dementia friendly health and social care systems, carers report the need for more appropriate and flexible support to improve their QoL. The importance of appropriate and tailored services has been noted previously (Oliveira et al., 2017) with such support having the ability to buffer caregiving burden and improve QoL. In addition, perceived quality of support appears to be more important than the tangible amount of support received for the well-being of family carers (Barrera, 1986; Wiles, 2003; Shurgot and Knight, 2005; Oliveira et al., 2016). Good levels of well-being are associated with satisfaction with services, good subjective health, and higher agency in older carers (Raivio et al., 2015). It could therefore be argued that issues surrounding tangible or perceived appropriate support are made worse due to the lack of attention that older carers receive from public health 
services in terms of interventions for themselves. This may be because the boundaries between services with regards to who should care for the carer means they sit uncomfortably within the structure of community based services in particular. Patients and their families may find enormous difficulty in gaining access to specific services and professionals may not always be trained to deal with the needs of the carer as well as the patient. Service provision can therefore often feel poor and unsuitable with families burdened with the main responsibility of care. We would argue that health care professionals need to further consider the carer within their service provision planning in order to improve QoL in this carer group.

In considering the theme of financial support, the carers in this study reported concerns over money and the financial impact of being a carer. This theme presented with less frequency than the other 2 key themes. However, the financial impact of caregiving runs through all the data implicitly in that additional funding could aid opportunities to enjoy life outside of the caregiving role and provide access to additional services. Alongside good-quality support, previous research also demonstrates that the financial situation of the older carer has an effect on their QoL (Schneider et al., 1999). Existing research has for decades alluded to the financial burden that caregiving in dementia places upon the family (e.g. Harrow et al., 2004; Ory et al., 1999) and this financial hardship is still experienced within this group of carers. With people living longer and spending more years of life at work, it is likely that older carers will be still working when the need to provide care for a family member arises. We would suggest that there needs to be more flexibility in care provision so that older carers can continue to work if they wish to do so. This is likely to have several benefits to the individual for example, increased satisfaction with life, keeping active and enabling independence and social activity. Moreover it may also be of benefit to societal economy as a whole in that working for longer is likely to improve a families overall financial situation, aiding financial independence.

This small scale study does have some potential limitations. As the qualitative data was collected via anonymous paper-based questionnaires, there was no opportunity to follow up the key themes in more depth with study participants. This is mitigated to some extent due to our large sample size but does present an opening for additional and more in-depth exploration of these themes in future work. Despite this limitation, the current study allowed for the identification of three common areas for 
improvement in QoL for this population of carers, which corroborate previous study findings.

\section{Conclusion}

Caring for someone with dementia can have a large impact on older family carers' QoL. The proportion of older individuals expected to look after their family members with dementia is increasing and little is known about how their QoL can be maintained or improved. This study suggests that older carers can most benefit from having more time away from their caregiving role, by health and social care services being more carer and dementia friendly, and by having better financial support. Older family carers need more respite that is suitable for their needs and meets their expectations. They need to be able to access more specialist services within a time frame that is suitable for them and be afforded the agency to financially manage their own affairs. Finally, we would argue that more public awareness of the contribution that family carers make to society is imperative to improve QoL in this carer group.

\section{References}

Ahn S, Hochhalter AK, Moudouni DKM, et al. (2012) Self-reported physical and mental health of older adults: the roles of caregiving and resources. Maturitas 71: 62-69.

Al-Zahrani R, Bashihab R, Ahmed AE, et al. (2015) The prevalence of psychological impact on caregivers of hospitalized patients: The forgotten part of the equation. Qatar Med J., 1. (accessed 10 January 2016).

Barrera MJ. (1986) Distinctions between social support concepts, measures, and models. Am J Commun Psychol. 14: 413-445.

Berkman LF, Cannuscio CC, Colditz GA, et al. (2004) Employment status, social ties, and caregivers' mental health. Social Science and Medicine 58: 1247-1256. 
Boer AGEM, van Lanschot JJB, Stalmeier PFM, et al. (2004) Is a single-item visual analogue scale as valid, reliable and responsive as multi-item scales in measuring quality of life? . Quality of Life Research 13: 311-320.

Braun V and Clarke V. (2006) Using thematic analysis in psychology. Qualitative Research in Psychology 3: 77-101.

Butterworth P, Pymont C, Rodgers B, et al. (2010) Factors that explain the poorer mental health of caregivers: results from a community survey of older Australians. Australian \& New Zealand Journal of Psychiatry 44: 616-624.

Carers Trust. (2011) Always On Call, Always Concerned : A Survey of the Experiences of Older Carers. Essex, UK: The Princess Royal Trust for Carers

Carers UK and Age UK. (2015) Caring into later life: The growing pressure on older carers. London: Carers UK.

Coen RF, O'Boyle CA, Coakley D, et al. (2002) Individual quality of life factors distinguishing low-burden and high-burden caregivers of dementia patients. Dementia \& Geriatric Cognitive Disorders 13: 164-170.

Covinsky KE, Newcomer RJ, Fox P, et al. (2003) Patient and caregiver characteristics associated with depression in caregivers of patients with dementia. J Gen Intern Med 18: 1006-1014.

Dilworth-Anderson P. (2015) Baby boomer caregiver and dementia caregiving: findings from the National Study of Caregiving. Age and Ageing 44: 300-306.

Ekwall AK, Sivberg B and Hallberg IR. (2005) Loneliness as a predictor of quality of life among older caregivers. J Adv Nurs 49: 23-32.

Fayers PM and Machin D. (2016) Quality of life: The assessment, analysis and interpretation of patient-reported outcomes, England: Wiley Blackwell.

Frith $\mathrm{H}$ and Gleeson K. (2004) Clothing and embodiment: men managing body image and appearance. Psychology of Men \& Masculinity 5: 40-48. 
Oliveira, Sousa \& Aubeeluck 2018 (accepted for publication - 'Dementia' SAGE on 4 Jul 2018)

Gallant MP and Connel CM. (1997) Predictors of decreased self-care among spouse caregivers of older adults with dementing illness. J Aging Health 9: 373-395.

Harrow B, Mahoney D, Mendelsohn A, et al. (2004) Variation in cost of informal caregiving and formal-service use for people with Alzheimer's disease. Am J Alzheimers Dis Other Demen. 19: 299-308.

Huang L. (2012) Effects of social networks on the health of family caregivers in later life: A cross-sectional study. Social Work. Michigan, USA: Michigan State University.

Jaremka LM, Andridge RR, Fagundes CP, et al. (2014) Pain, Depression, and Fatigue: Loneliness as a Longitudinal Risk Factor. Health Psychology 33: 948957.

Kane M and Terry G. (2015) Dementia 2015: Aiming higher to transform lives. London: Alzheimer's Society.

Lautenschlager N. (2013) Physical and mental health of ageing carers. 16th International Congress of the International Psychogeriatric Association. Seoul, South Korea: Cambridge University Press, S16.

Lavela SL and Ather N. (2010) Psychological health in older adult spousal caregivers of older adults. Chronic IIIn 6: 67-80.

Macbeth D. (2001) On "Reflexivity" in qualitative research: Two readings and a Third. Qualitative Enquiry 7: 35-68.

Moon $\mathrm{H}$ and Dilworth-Anderson P. (2015) Baby boomer caregiver and dementia caregiving: findings from the National Study of Caregiving. Age Ageing 44: 300-306.

Neri AL, Yassuda MS, Fortes-Burgos AC, et al. (2012) Relationships between gender, age, family conditions, physical and mental health, and social isolation of elderly caregivers. Int Psychogeriatr 24: 472-483.

NHS. (2015) 2014/15 National Tariff Payment System. 
Oliveira D, Neri A and Delboux M. (2016) Lack of anticipated support for care for community-dwelling older adults. Rev Bras Enferm 69: 1-10.

Oliveira D, Vass C and Aubeeluck A. (2015) Ageing and quality of life in family carers of people with dementia being cared for at home: a literature review. Quality in Primary Care 23: 18-30.

Oliveira D, Vass C and Aubeeluck A. (2017) Quality of life on the views of older family carers of people with dementia. Dementia 0: 1-20.

Ory M, Hoffman R, Yee J, et al. (1999) Prevalence and impact of caregiving: a detailed comparison between dementia and nondementia caregivers. Gerontologist 39: 177-185.

Prince M, Knapp M, Guerchet M, et al. (2014) Dementia UK: Update. London: Alzheimer's Society, 1-136.

Quinn C, Clare L and Woods B. (2009) The impact of the quality of relationship on the experiences and wellbeing of caregivers of people with dementia: a systematic review. Aging Ment Health. 13: 143-154.

Raivio MM, Laakkonen ML and Pitkala KH. (2015) Psychological well-being of spousal caregivers of persons with Alzheimer's disease and associated factors. European Geriatric Medicine 6: 128-133.

Rozario PA, Morrow-Howell N and Hinterlong JE. (2004) Role Enhancement or Role Strain: Assessing the Impact of Multiple Productive Roles on Older Caregiver Well-Being. Research on Aging 26: 413-428.

Schneider J, Murray J, Banerjee S, et al. (1999) Eurocare: A cross-sectional study of co-resident spouse carers for people with Alzheimer's disease: I-Factors associated with carer burden. International Journal of Geriatric Psychiatry 14: 651-661.

Shurgot GR and Knight BG. (2005) Influence of Neuroticism, Ethnicity, Familism, and Social Support on Perceived Burden in Dementia Caregivers: Pilot Test of the 
Oliveira, Sousa \& Aubeeluck 2018 (accepted for publication - 'Dementia' SAGE on 4 Jul 2018)

Transactional Stress and Social Support Model. Journal of Gerontology: Psychological Sciences 60B: S331-P334.

Steptoe A, Shankar A and Snorri R. (2015) The emotional well-being of older carers. In: University College London (UCL): Department of Epidemiology and Public Health (ed). London, UK.

Vitaliano PP, Murphy M, Young HM, et al. (2011) Does caring for a spouse with dementia promote cognitive decline? A hypothesis and proposed mechanisms. J Am Geriatr Soc. 59: 900-908.

White C. (2013) Census Analysis - Unpaid care in England and Wales, 2011 in comparison with 2001. In: ONS (ed). Newport, Wales: Crown.

Wiles J. (2003) Informal caregivers' experiences of formal support in a changing context. Health \& Social Care in the Community 11: 189-207.

World Medical Association. (2013) Declaration of Helsinki. Ethical principles for medical research involving human subjects. JAMA 310: 2191-2194. 